\title{
Qualidade de vida e os métodos de comunicação alaríngea: uma revisão de escopo
}

\author{
Quality of life and alaryngeal communication methods: a scoping review \\ Calidad de vida y métodos de comunicación alaríngea: una revisión del alcance
}

\author{
Laryssa da Cunha Santos \\ ORCID: https://orcid.org/0000-0002-4787-5985 \\ Universidade Federal de Sergipe; Brasil \\ E-mail: laryssapixton@gmail.com \\ Thaynara Andrade Santos \\ ORCID: https://orcid.org/0000-0001-5970-3358 \\ Universidade Federal de Sergipe; Brasil \\ E-mail: thaynarasantos152@yahoo.com \\ Kelly da Silva \\ ORCID: https://orcid.org/0000-0002-9193-7282 \\ Universidade Federal de Sergipe; Brasil \\ E-mail:kelly.silva@academico.ufs.br \\ Ariane Damasceno Pellicani \\ ORCID: https://orcid.org/0000-0003-0390-9175 \\ Universidade Federal de Sergipe; Brasil \\ E-mail:adpellicani@academico.ufs.br
}

\begin{abstract}
Resumo
Objetivo: apresentar um mapeamento dos estudos envolvendo a qualidade de vida de laringectomizados totais quanto aos métodos de comunicação alaríngea e instrumentos utilizados. Método: A pergunta a ser respondida é: "Quais os instrumentos, validados e confiáveis psicometricamente, utilizados para avaliar a qualidade de vida relacionada a comunicação alaríngea de laringectomizados totais?". Para responder a essa pergunta foram definidos os seguintes critérios de elegibilidade, de acordo com o acrônimo: P- População - Laringectomizados totais, C- Conceito - Outra forma de comunicação alaríngea, C- Contexto- Qualidade de vida após laringectomia total. Após a definição dos critérios de elegibilidade, consulta à bases de dados com os descritores DeCS/MeSH e Emtree: "Laryngectomy, Quality of Life, Speech, Alaryngeal", combinados com o operador boleano “AND” e para os idiomas português, inglês e espanhol. Resultados: foram obtidos 238 textos científicos, sendo 4 artigos na Lilacs, 74 na PubMed, 21 na Embase, 92 na Scopus, 42 na Web of Science, zero (0) na OpenGrey, dois (2) no Google Acadêmico e três (3) na BDTD (Biblioteca Digital Brasileira de Teses e Dissertações). Conclussão: Os instrumentos mais utilizados para avaliar a qualidade de vida em laringectomizados totais são: Qualidade de Vida em Voz (QVV), seguido do University of Washington Quality of Life Questionnaire (UW-QOL), e dos protocolos EORTC Quality of Life Questionnaire (EORTC QLQ-C30), Instrumento Qualidade De Vida da Universidade De Michigan Head And Neck (HNQOL), Questionário de Qualidade de Vida -SF-36.

Palavras-chave: Qualidade de vida; Protocolos de autoavaliação; Laringectomizados totais; Voz traqueoesofágica; Comunicação alaríngea.
\end{abstract}

\begin{abstract}
Objective: To present a mapping of studies involving the quality of life of the total laryngectomees regarding the laryngeal communication methods and instruments used. Method: The question to be answered is: "Which instruments, validated and psychometrically reliable, are used to assess the quality of life-related to laryngeal communication in total laryngectomees?". To answer this question, the following eligibility criteria were defined, according to the acronym: P- Population - Total laryngectomy patients, C- Concept - Another form of laryngeal communication, C- Context- Quality of life after total laryngectomy. After defining the eligibility criteria, consult the databases with the DeCS/MeSH and Emtree descriptors: "Laryngectomy, Quality of Life, Speech, Alaryngeal", combined with the Boolean operator "AND” and for Portuguese, English, and Portuguese. Spanish. Results: 238 scientific texts were obtained, 4 articles in Lilacs, 74 in PubMed, 21 in Embase, 92 in Scopus, 42 in Web of Science, zero (0) in OpenGrey, two (2) in Google Scholar, and three (3) at the BDTD (Brazilian Digital Library of Theses and Dissertations). Conclusion: The instruments most used to assess the quality of life in total laryngectomees are: Quality of Life in Voice (QVV), followed by the University of Washington Quality of Life Questionnaire (UW-QOL), and the EORTC Quality of Life Questionnaire protocols ( EORTC QLQ-C30), the University of Michigan Head And Neck Quality of Life Instrument (HNQOL), Quality of Life Questionnaire -SF-36.
\end{abstract}

Keywords: Quality of life; Self-assessment protocols; Total laryngectomy; Tracheoesophageal voice; Laryngeal communication. 


\begin{abstract}
Resumen
Objetivo: Presentar un mapeo de estudios que involucran la calidad de vida de los laringectomizados totales en cuanto a los métodos e instrumentos de comunicación laríngea utilizados. Método: La pregunta a responder es: “¿Qué instrumentos, validados y psicométricamente confiables, se utilizan para evaluar la calidad de vida relacionada con la comunicación laríngea en laringectomizados totales?". Para responder a esta pregunta, se definieron los siguientes criterios de elegibilidad, según la sigla: P- Población - Pacientes laringectomizados totales, C- Concepto - Otra forma de comunicación laríngea, C- Contexto- Calidad de vida después de la laringectomía total. Después de definir los criterios de elegibilidad, consulte las bases de datos con los descriptores DeCS/MeSH y Emtree: "Laryngectomy, Quality of Life, Speech, Alaryngeal", combinado con el operador booleano "AND" y para portugués, inglés y portugués. Español. Resultados: se obtuvieron 238 textos científicos, 4 artículos en Lilacs, 74 en PubMed, 21 en Embase, 92 en Scopus, 42 en Web of Science, cero (0) en OpenGrey, dos (2) en Google Scholar y tres (3) en la BDTD (Biblioteca Digital Brasileña de Tesis y Disertaciones). Conclusión: Los instrumentos más utilizados para evaluar la calidad de vida en laringectomizados totales son: Quality of Life in Voice (QVV), seguido del Cuestionario de Calidad de Vida de la Universidad de Washington (UW-QOL), y los protocolos del Cuestionario de Calidad de Vida de la EORTC (EORTC QLQ-C30), el Instrumento de Calidad de Vida de Cabeza y Cuello de la Universidad de Michigan (HNQOL), Cuestionario de Calidad de Vida -SF-36.

Palabras clave: Calidad de vida; Protocolos de autoevaluación; Laringectomía total; Voz traqueoesofágica; Comunicación laríngea.
\end{abstract}

\title{
1. Introdução
}

A população laringectomizada total tem como principal impacto pós-cirúrgico a ausência da emissão vocal e o comprometimento da comunicação oral. O uso de métodos alaríngeos de comunicação oral possibilitam ao indivíduo o retorno e melhoria de sua comunicação, o que pode impactar em ganhos nos âmbitos pessoais, sociais e profissionais.

Apesar desse retorno à comunicação oral alaríngea, cada método de comunicação possui seus impactos positivos e negativos. Um estudo (Choi et al., 2001), resssalta que a vantagem da voz esofágia é que não necessita de uma nova cirurgia e nem de uso de nenhum aparelho. Os autores colocam como desvantagem a ausência de uma intensidade vocal satisfatória em uma conversação realizada em ambiente ruidoso. Blom et al. (1986) ainda ressaltam que é reduzida a porcentagem de pacientes que conseguem desenvolver a voz esofágica de forma eficaz.

Um estudo (Soto et al., 2005) ressalta que na voz traqueoesofágica, as principais desvantagens são as trocas frequentemente e o alto custo, mas em contrapartida as suas vantagens são a rápida aquisição da voz, o uso do ar pulmonar, o índice de sucesso de $90 \%$ e a boa qualidade vocal. É o método de comunicação alaríngea que mais se aproxima da voz laríngea (Medeiros, 2008) e a mais utilizada em países desenvolvidos. Apresenta um alto custo, visto a necessidade de trocas frequentes e a exigência de higiene impecável (Ghirardi, 2007).

A comunicação por meio da laringe eletrônica possibilta a saída do silêncio involuntário do laringectomizado total, entretanto, alguns aspectos da voz podem promover impacto negativo ao paciente, tais como, voz robótica, expressão vocal reduzida, limitação do uso das mãos e gestos, entre outros. O estudo Bloom, Singer, Hamaker25 coloca como uma vantagem da laringe eletrônica ser o método mais facil para os pacientes e, como desvantagem, a questão da voz ser mais artificial e monótona.

Dessa forma, é possível observar que cada método de comunicação tem impactos positivos e negativos (Behlau, 2005), o que pode impactar na qualidade de vida do laringectomizado. Estudos têm demonstrado os efeitos da comunicação alaríngea na qualidade de vida do laringectomizado total, entretanto, não há um consenso referente a qual protocolo validado a ser utilizado e qual o método que melhor beneficia a qualidade de vida do indivíduo.

O presente estudo tem por objetivo apresentar um mapeamento dos estudos envolvendo a qualidade de vida de laringectomizados totais quanto aos métodos de comunicação alaríngea e instrumentos utilizados. 


\section{Metodologia}

Esta revisão possui registro Open Science com DOI 10.17605/OSF.IO/FTKYQ e foi elaborada de acordo com metodologia de análise de escopo, recomendada pelo Instituto Joanna Briggs (Santos et al., 2018).

Pretende-se apresentar um mapeamento dos estudos envolvendo a qualidade de vida de laringectomizados totais quanto aos métodos de comunicação alaríngea. E também, fornecer uma visão descritiva dos estudos revisados, sem avalia-los criticamente (CONITEC, 2018), entretanto, seguindo protocolos recomendados na literatura, como o PRISMA.

A pergunta a ser respondida por meio desta revisão é: "Quais os instrumentos, validados e confiáveis psicometricamente, utilizados para avaliar a qualidade de vida relacionada a comunicação alaríngea de laringectomizados totais?". Para responder a essa pergunta foram definidos os seguintes critérios de elegibilidade, de acordo com o acrônimo PCC:

P- População - Laringectomizados totais

C- Conceito - Outra forma de comunicação alaríngea

C- Contexto- Qualidade de vida após laringectomia total

Foram incluídos estudos que utilizassem a população de pacientes laringectomizados totais, sem distinção de gênero, idade, ano de publicação e tipos de estudos, falantes por meio da prótese traqueoesofágica, voz esofágica e laringe eletrônica e que tiveram a qualidade de vida mensurada por protocolo validado. A inclusão restringiu-se aos artigos redigidos em espanhol, inglês ou português de desenho metodológico distintos.

Foram excluídos estudos que englobassem pacientes submetidos a laringectomias totais ampliadas (por exemplo: exérese de terço superior esôfago, faringolaringectomia, glossofaringolaringectomias, entre outras) ou laringectomias parciais. Também não foram aceitos artigos de revisões de literatura, resumo em congressos/conferências e estudos qualitativos.

A revisão foi realizada no primeiro semestre de 2021 por meio do acesso às bases de dados Lilacs (Literatura LatinoAmericana e do Caribe em Ciências da Saúde), PubMed, Embase, Scopus, Web of Science, OpenGrey, Google acadêmico e BDTD (Biblioteca Digital Brasileira de Teses e Dissertações). Não foi realizado contato adicional com autores das publicações analisadas.

Os descritores empregados para a seleção dos estudos, obtidos junto ao DeCS/MeSH (Descritores em Ciências da Saúde) e Emtree, foram: "Laryngectomy, Quality of Life, Speech, Alaryngeal", combinados com o operador boleano "AND" para os idiomas português, inglês e espanhol.

Para a seleção dos artigos, primeiramente, foi realizada a busca em bases de dados com as estratégias descritas anteriormente; seleção do material publicado de acordo com os critérios de inclusão e exclusão através da leitura de título e resumo; aplicação dos critérios de inclusão e exclusão na leitura completa do material selecionado; leitura do texto completo e extração os dados de relevância para a presente revisão.

A seleção dos artigos e coleta de dados foi realizada por duas revisoras previamente calibradas, assessoradas por uma terceira revisora para os casos de discrepância. Foram realizadas reuniões para o encontro de um consenso dos títulos e resumos selecionados.

Posteriormente, foi realizada a leitura na íntegra dos artigos selecionados e analisados os critérios de elegibilidade. Esta etapa seguiu com a participação de duas revisoras calibradas e um terceiro revisor com expertise na área para os casos de discordância na seleção. Novamente, foram realizadas reuniões para discutir os artigos selecionados e reduzir a possibilidade de viés. Foram convidadas a participar deste estudo outras duas pesquisadoras fonoaudiólogas com expertise em revisões de literatura visando o subsídio de uma adequada condução da metodologia, extração e análise dos dados.

As variáveis extraídas foram: base de dados, autores, ano de publicação, revista, idioma de publicação, país, instituição. Também foram analisados os resultados das aplicações dos protocolos de qualidade de vida na comunicação 
alaríngea após a laringectomia total. Foram analisados o protocolo utilizado, método de comunicação alaríngea, número de participantes, gênero, domínios dos protocolos de qualidade de vida.

O gerenciador de referências Mendeley ${ }^{\circledR}$ foi utilizado como instrumento para organização dos artigos. Os dados coletados foram tabulados em planilha Excel@e duplamente verificados pelos pesquisadores para a apresentação dos resultados. A apresentação dos resultados seguiu as orientações PRISMA-ScR.

A Figura 1 apresenta o processo de busca e seleção dos artigos conforme os critérios estabelecidos até a confecção do banco de dados final.

Figura 1: Fluxograma de apresentação das fases de seleção dos estudos analisados.
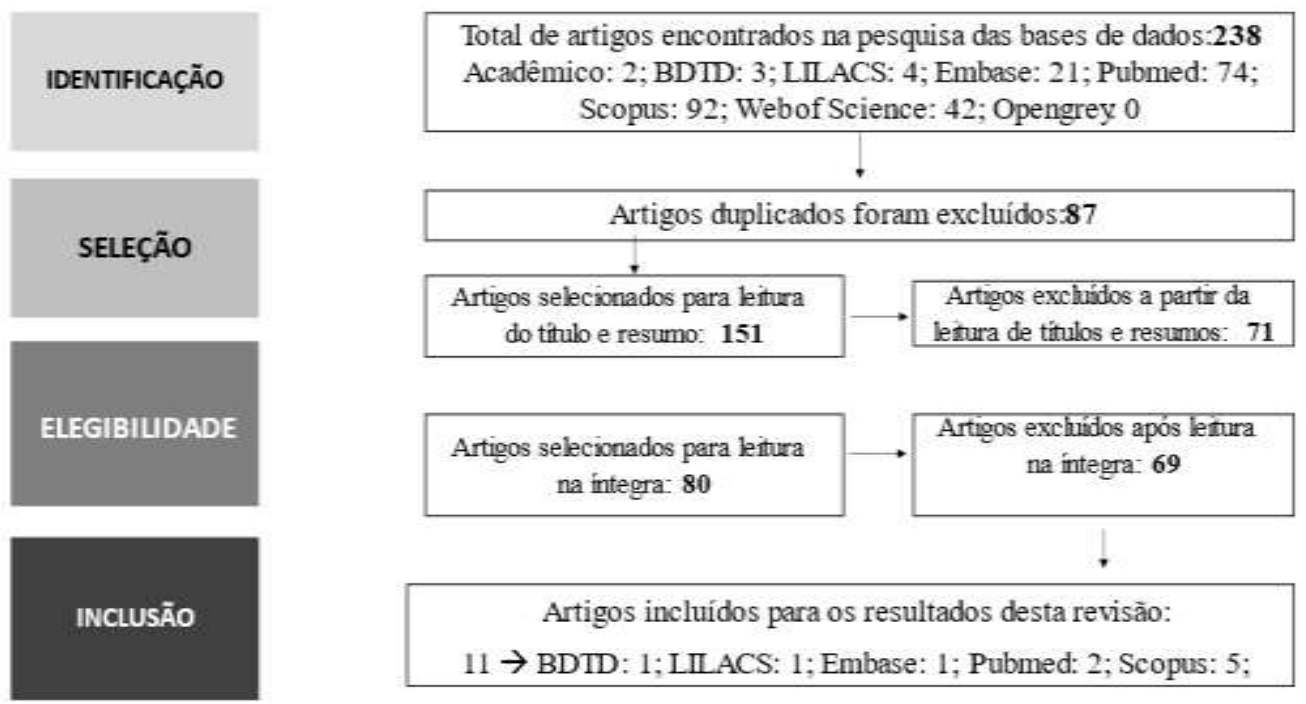

Fonte: Autores.

\section{Resultados}

Os estudos incluídos foram publicados entre 2000 a 2017. A Tabela 1 apresenta o mapeamento dos estudos incluídos. 
Tabela 1: Mapeamento dos artigos selecionados.

\begin{tabular}{|c|c|c|c|c|c|c|c|c|c|}
\hline $\begin{array}{c}\text { Base de } \\
\text { dados }\end{array}$ & Título do artigo & Autores & Ano & $\begin{array}{c}\text { Jornal de } \\
\text { publicação }\end{array}$ & Idioma & $\begin{array}{c}\mathbf{N}^{0} \\
\text { participantes } \\
\end{array}$ & Instituição & Cidade & País \\
\hline SCOPUS & $\begin{array}{l}\text { Assessing self- } \\
\text { reported measures } \\
\text { of voice disability } \\
\text { in } \\
\text { tracheoesophageal } \\
\text { speakers }\end{array}$ & $\begin{array}{l}\text { DAY, A. M.; } \\
\text { DOYLE, Philip } \\
\text { C }\end{array}$ & 2010 & $\begin{array}{c}\text { Journal of } \\
\text { otolaryngology- } \\
\text { head \& neck } \\
\text { surgery }\end{array}$ & Inglês & 30 & $\begin{array}{l}\text { Western } \\
\text { University }\end{array}$ & Ontario & Canadá \\
\hline PUBMED & $\begin{array}{l}\text { Auditory- } \\
\text { perceptual scaling } \\
\text { and quality of life } \\
\text { in } \\
\text { tracheoesophageal } \\
\text { speakers. }\end{array}$ & $\begin{array}{l}\text { EADIE, Tanya } \\
\text { L.; DOYLE, } \\
\text { Philip C }\end{array}$ & 2004 & The Laryngoscope & Inglês & 43 & $\begin{array}{l}\text { University of } \\
\text { Washington }\end{array}$ & Seattle & EUA \\
\hline SCOPUS & $\begin{array}{l}\text { Auditory- } \\
\text { perceptual speech } \\
\text { outcomes and } \\
\text { quality of life after } \\
\text { total laryngectomy }\end{array}$ & $\begin{array}{c}\text { EADIE, Tanya } \\
\text { L. et al. }\end{array}$ & 2013 & $\begin{array}{l}\text { Otolaryngology-- } \\
\text { Head and Neck } \\
\text { Surgery, }\end{array}$ & Inglês & 25 & $\begin{array}{l}\text { University of } \\
\text { Washington }\end{array}$ & Seattle & EUA \\
\hline PUBMED & $\begin{array}{l}\text { Communication } \\
\text { after } \\
\text { laryngectomy: an } \\
\text { assessment of } \\
\text { quality of life. }\end{array}$ & $\begin{array}{l}\text { CARR, Michele } \\
\text { M. et al. }\end{array}$ & 2000 & $\begin{array}{c}\text { Otolaryngology- } \\
\text { Head and Neck } \\
\text { Surgery }\end{array}$ & Inglês & 50 & $\begin{array}{c}\text { Queen } \\
\text { Elizabeth II } \\
\text { Health } \\
\text { Sciences } \\
\text { Centre }\end{array}$ & Halifax & Canadá \\
\hline WEB & $\begin{array}{c}\text { Coping and } \\
\text { Quality of Life aer } \\
\text { Total } \\
\text { Laryngectomy }\end{array}$ & $\begin{array}{l}\text { EADIE, Tanya } \\
\text { L.; BOWKER, } \\
\text { Brianne C }\end{array}$ & 2012 & $\begin{array}{c}\text { Otolaryngology-- } \\
\text { Head and Neck } \\
\text { Surgery }\end{array}$ & Inglês & 77 & $\begin{array}{l}\text { University of } \\
\text { Washington }\end{array}$ & Seattle & EUA \\
\hline EMBASE & $\begin{array}{l}\text { Generic health- } \\
\text { related quality of } \\
\text { life amongst } \\
\text { patients employing } \\
\text { different voice } \\
\text { restoration } \\
\text { methods following } \\
\text { total laryngectomy }\end{array}$ & $\begin{array}{c}\text { FARRAND, } \\
\text { Paul; DUNCAN, } \\
\text { Fiona. }\end{array}$ & 2007 & $\begin{array}{l}\text { Psychology, health } \\
\text { \& medicine }\end{array}$ & Inglês & 310 & $\begin{array}{l}\text { University of } \\
\text { Plymouth, } \\
\text { Exeter } \\
\text { Campus }\end{array}$ & Exter & $\begin{array}{l}\text { Reino } \\
\text { Unido }\end{array}$ \\
\hline SCOPUS & $\begin{array}{l}\text { Speech } \\
\text { intelligibility, } \\
\text { acceptability, and } \\
\text { communication- } \\
\text { related quality of } \\
\text { life in Chinese } \\
\text { alaryngeal } \\
\text { Speakers }\end{array}$ & $\begin{array}{c}\text { LAW, Ida K.-Y.; } \\
\text { MA, Estella P.- } \\
\text { M.; YIU, Edwin } \\
\text { M.-L. }\end{array}$ & 2009 & $\begin{array}{l}\text { Archives of } \\
\text { Otolaryngology- } \\
\text { Head \& Neck } \\
\text { Surgery }\end{array}$ & Inglês & 49 & $\begin{array}{l}\text { University of } \\
\text { Hong Kong, }\end{array}$ & Hong Kong & China \\
\hline SCOPUS & $\begin{array}{l}\text { The influence of } \\
\text { electrolarynx use } \\
\text { on } \\
\text { postlaryngectomy } \\
\text { voice-related } \\
\text { quality of life }\end{array}$ & $\begin{array}{c}\text { COX, Steven R.; } \\
\text { DOYLE, Philip } \\
\text { C }\end{array}$ & 2014 & $\begin{array}{c}\text { Otolaryngology-- } \\
\text { Head and Neck } \\
\text { Surgery }\end{array}$ & Inglês & 40 & $\begin{array}{l}\text { Western } \\
\text { University }\end{array}$ & Ontario & Canadá \\
\hline SCOPUS & $\begin{array}{l}\text { Voice-related } \\
\text { quality of life (V- } \\
\text { RQOL) outcomes } \\
\text { in laryngectomees }\end{array}$ & $\begin{array}{l}\text { MOUKARBEL, } \\
\text { Roger V. et al }\end{array}$ & 2011 & Head \& neck & Inglês & 75 & $\begin{array}{l}\text { Western } \\
\text { University }\end{array}$ & Ontario & Canadá \\
\hline LILACS & $\begin{array}{l}\text { Voz e qualidade de } \\
\text { vida de } \\
\text { laringectomizados } \\
\text { totais: um estudo } \\
\text { comparativo } \\
\end{array}$ & $\begin{array}{l}\text { CORREIA, ME; } \\
\text { VIANNA, KMP; } \\
\text { GHIRARDI, } \\
\text { ACAM. }\end{array}$ & 2016 & Revista CEFAC & Português & 11 & $\begin{array}{l}\text { Universidade } \\
\text { Federal de } \\
\text { Santa } \\
\text { Catarina } \\
\text { (UFSC) } \\
\end{array}$ & Florianópolis & Brasil \\
\hline
\end{tabular}

Fonte: Autores.

A Tabela 2 apresentam as características dos artigos incluídos, sendo possível observar um maior quantitativo de publicações do Canadá. 
Tabela 2: Características das publicações analisadas.

\begin{tabular}{|c|c|c|}
\hline Variável & $\mathbf{N}^{\circ}$ & $\%$ \\
\hline \multicolumn{3}{|l|}{ Ano } \\
\hline $2000-2004$ & 2 & 18,18 \\
\hline 2005-2009 & 2 & 18,18 \\
\hline 2010-2014 & 5 & 45,45 \\
\hline $2015-2020$ & 2 & 18,18 \\
\hline \multicolumn{3}{|c|}{ Publicação } \\
\hline Journal Of Otolaryngology-Head \& Neck Surgery & 1 & 9,09 \\
\hline The Laryngoscope & 1 & 9,09 \\
\hline Otolaryngology--Head and Neck Surgery & 4 & 36,36 \\
\hline Psychology, Health \& Medicine & 1 & 9,09 \\
\hline BDTI & 1 & 9,09 \\
\hline Archives of Otolaryngology-Head \& Neck Surgery & 1 & 9,09 \\
\hline Head \& Neck & 1 & 9,09 \\
\hline Revista CEFAC & 1 & 9,09 \\
\hline \multicolumn{3}{|c|}{ País de Origem } \\
\hline Canada & 4 & 36,36 \\
\hline EUA & 3 & 27,27 \\
\hline Brasil & 2 & 18,18 \\
\hline China & 1 & 9,09 \\
\hline Reino Unido & 1 & 9,09 \\
\hline
\end{tabular}

Fonte: Autores.

O Quadro 1 apresenta o mapeamento de acordo com o método de comunicação utilizado no estudo, protocolo de qualidade de vida e desfecho. Um total de seis estudos utilizaram o protocolo QVV para mensurar a qualidade de vida nos laringectomizados totais.

Quadro 1: Descritiva da identificação, objetivo e metodologia dos artigos incluídos no presente estudo.

\begin{tabular}{|c|c|c|c|c|}
\hline $\begin{array}{l}\text { Autores (ano) } \\
\text { Revista } \\
N^{0} \text {. Vol }\end{array}$ & Métodos & $\begin{array}{l}\text { Método de } \\
\text { comunicação } \\
\text { oral }\end{array}$ & $\begin{array}{l}\text { Protocolo QV } \\
\text { utilizado }\end{array}$ & Desfecho \\
\hline $\begin{array}{c}\text { Day, AM.; Doyle, PC. (2010) }{ }^{14} \\
\text { Journal of otolaryngology } \\
\text { v. } 39, \text { n. } 6\end{array}$ & $\begin{array}{c}\text { Trinta (15 homens e } 15 \text { mulheres); } \\
65 \text { anos (variação } 51-75 \text { anos) para homens e mulheres; } \\
\text { Laringectomizados totais } \\
22 \text { participantes (11 homens, } 11 \text { mulheres) receberam radioterapia, } 4 \text { de } \\
\text { ambos os grupos não; } \\
\text { Tempo médio da punção da PTE = } 88 \text { meses ( } 7 \text { anos, } 4 \text { meses) } \\
\text { Analise em função do gênero }\end{array}$ & PTE & V-RQOL & $\begin{array}{l}\text { Homens } \\
\text { melhor QV } \\
\text { com PTE que } \\
\text { mulheres }\end{array}$ \\
\hline $\begin{array}{c}\text { Eadie, TL. Doyle PC. }(2004)^{15} \\
\text { The Laryngoscope } \\
\text { v. } 114 \text {, n. } 4\end{array}$ & $\begin{array}{c}\text { Grupo 1: PTE - } 28 \text { laringectomizados totais (LT), idade: } 42-82 \text { anos } \\
\text { para homens e 57-72 anos para mulheres } \\
\text { Grupo 2: } 15 \text { alunos de graduação- ouvintes das vozes LT, idade: } 25,87 \\
\text { anos } \\
\text { Análise da qualidade de vida x analise perceptivo-auditiva }\end{array}$ & PTE & HNQOL. 10 & $\begin{array}{l}\text { PTE com boa } \\
\text { qualidade de } \\
\text { vida associada } \\
\text { aos domínios } \\
\text { do protocolo }\end{array}$ \\
\hline $\begin{array}{l}\text { Eadie TL, Day AMB, Sawin } \\
\text { DE, Lamvik K, Doyle PC. } \\
(2013)^{18} \\
\text { Otolaryngology--Head and } \\
\text { Neck Surgery } \\
\text { v. } 148, \text { n. } 1\end{array}$ & $\begin{array}{c}\text { Grupo 1: } 25 \text { laringectomizados totais. Média idade } 63,3 \text { anos (64,8 anos } \\
\text { para homens e 59,2 anos para mulheres) } \\
\text { Grupo 2: } 33 \text { ouvintes experientes } \\
\text { Análise da qualidade de vida x inteligibilidade de fala, aceitabilidade }\end{array}$ & PTE, VE e LE & UW-QOL & $\begin{array}{l}\mathrm{VE}>\mathrm{PTE}>\mathrm{LE} \\
\text { na qualidade } \\
\text { de vida para a } \\
\quad \text { fala. }\end{array}$ \\
\hline $\begin{array}{l}\text { Carr MM, Schmidbauer JA, Ma } \\
\text { C, Majaess L. (2000) }{ }^{19} \\
\text { Otolaryngology-Head and Neck } \\
\text { Surgery } \\
\text { v.122 }\end{array}$ & $\begin{array}{c}\begin{array}{c}34 \text { laringe eletrônica, } \\
11 \text { voz esofágica } \\
5 \text { PTE }\end{array} \\
\text { Análise da qualidade de vida x métodos de comunicação alaríngea }\end{array}$ & PTE, VE e LE & EORTC & $\begin{array}{l}\text { VE }>\text { LE }>\text { PTE } \\
\text { na qualidade } \\
\text { de vida para a } \\
\text { função de } \\
\text { fala. }\end{array}$ \\
\hline
\end{tabular}




\begin{tabular}{|c|c|c|c|c|}
\hline $\begin{array}{l}\text { Eadie TL, Bowker BC }(2012)^{20} \\
\text { Otolaryngology--Head and } \\
\text { Neck Surgery } \\
\text { v.146 }\end{array}$ & $\begin{array}{c}67 \text { pacientes com laringectomia total: } 20 \text { LE, } 35 \text { PTE, } 7 \text { VE e } 5 \text { com } \\
\text { escrita. } \\
51 \text { homens, } 16 \text { mulheres } \\
\text { Média de } 63 \text { anos idade (faixa, } 44-89 \text { anos) } \\
\text { Média } 84 \text { meses após laringectomia; } \\
\text { Análise da estratégia de enfrentamento x fatores oncológicos e } \\
\text { qualidade de vida. } \\
\end{array}$ & LE, PTE, VE & $\begin{array}{l}\text { UW-QOL } \\
\text { V-RQOL }\end{array}$ & $\begin{array}{l}\text { UW-QOL: } \\
\text { qualidade de } \\
\quad \text { vida } \\
\text { moderado. } \\
\text { QVV: LE>VE } \\
\quad>\text { PTE }\end{array}$ \\
\hline $\begin{array}{l}\text { Farrand P, Duncan F }(2007)^{21} \\
\text { Psychology, health \& medicine } \\
\text { v. } 12\end{array}$ & $\begin{array}{c}\text { Pacientes com laringectomia total; } \\
\text { Grupo controle: } 89 \text { falantes laringe } \\
\text { Grupo estudo: } 147 \text { pacientes falantes por PTE, } 42 \text { falantes de VE, } 37 \\
\text { falantes por LE } \\
\text { Ao todo foram } 202 \text { homens e } 108 \text { mulheres } \\
\text { Análise da qualidade de vida x métodos de comunicação alaríngea }\end{array}$ & PTE, VE e LE & SF-36 & $\begin{array}{l}\text { PTE }>\text { VE } \\
>\text { LE na } \\
\text { percepção } \\
\text { geral de } \\
\text { saúde. }\end{array}$ \\
\hline $\begin{array}{l}\text { Almeida, ALC (2017) }{ }^{16} \\
\text { Teses e Dissertações Unicamp }\end{array}$ & $\begin{array}{l}20 \text { laringectomizados totais } \\
\text { Pré e pós colocação de PTE }\end{array}$ & PTE & $\begin{array}{l}\text { V-RQOL } \\
\text { UWQOL }\end{array}$ & $\begin{array}{l}\text { UW-QOL e } \\
\text { QVV bons } \\
\text { índices de } \\
\text { qualidade de } \\
\text { vida. }\end{array}$ \\
\hline $\begin{array}{l}\text { Law IKY, Ma EPM, Yiu EML } \\
(2009)^{22} \\
\text { Archives of Otolaryngology- } \\
\text { Head \& Neck Surgery } \\
\text { v.135 }\end{array}$ & $\begin{array}{l}49 \text { pacientes LT, com idades entre } 40 \text { e } 77 \text { e com um período pós- } \\
\text { operatório de } 6 \text { a } 194 \text { meses foram incluídos na análise final, sendo } 45 \\
\text { homens e } 4 \text { mulheres; } \\
\text { Análise da qualidade de vida x métodos de comunicação alaríngea }\end{array}$ & $\begin{array}{l}\text { LE, PTE, VE } \\
\text { e dispositivo } \\
\text { pneumatico }\end{array}$ & $\begin{array}{l}\text { Questionário } \\
\text { de } \\
\text { Participação } \\
\text { Após } \\
\text { Laringectomia } \\
\text { (CAPAL) }\end{array}$ & $\begin{array}{l}\text { QV } \\
\mathrm{LE}>\mathrm{VE}>\mathrm{PTE} \\
\text {, apesar da } \\
\text { pobre } \\
\text { inteligibilidad } \\
\text { e de fala. } \\
\end{array}$ \\
\hline $\begin{array}{l}\text { Cox SR, Doyle PC }(2014)^{17} \\
\text { Otolaryngology--Head and } \\
\text { Neck Surgery } \\
\text { v. } 150\end{array}$ & $\begin{array}{l}40 \text { laringectomizados adultos ( } 25 \text { homens, } 15 \text { mulheres) } \\
\text { Uso exclusivo da laringe eletrônica } \\
\text { Idade: } 41 \text { a } 83 \text { anos (média, } 62,4 \text { anos) }\end{array}$ & $\mathrm{LE}$ & V-RQOL & $\begin{array}{l}\text { Homens } \\
\text { melhor } \\
\text { qualidade de } \\
\text { vida que } \\
\text { mulheres } \\
\end{array}$ \\
\hline $\begin{array}{l}\text { Moukarbel, R }(2011)^{23} \\
\text { Head \& neck } \\
\text { v.33 }\end{array}$ & $\begin{array}{c}75 \text { pacientes (51 homens, } 24 \text { mulheres), } \\
\text { Idade média de } 64,1 \text { anos } \\
18 \text { LE, } 15 \text { VE e } 42 \text { PTE. } \\
\text { Tempo médio após cirurgia foi de } 47,126 \text { e } 76 \text { meses para o LE, VE, e } \\
\text { grupos PTE, respectivamente } \\
\text { Análise da qualidade de vida x métodos de comunicação alaríngea }\end{array}$ & LE, PTE, VE & V-RQOL & $\begin{array}{l}\text { QV PTE=VE } \\
\text { LE melhor a } \\
\text { longo prazo }\end{array}$ \\
\hline $\begin{array}{l}\text { Correia ME. }(2016)^{8} \\
\text { Revista CEFAC } \\
\text { v.18 }\end{array}$ & $\begin{array}{l}11 \text { pacientes laringectomizados totais, } 8 \text { LE e } 3 \text { por PTE } \\
\text { Idade entre } 43 \text { e } 84 \text { anos (média } 65+10,5 \text { anos); } \\
\qquad 2 \text { mulheres e } 9 \text { homens } \\
\text { empo de cirurgia entre } 18 \text { e } 204 \text { meses (média } 75,4+66,8 \text { ); } \\
\text { Análise da qualidade de vida x métodos de comunicação alaríngea }\end{array}$ & LE e PTE & V-RQOL & QV PTE>LE \\
\hline
\end{tabular}

QV: qualidade de vida; PTE: prótese traqueoesofágica; VE: voz esofágica; LE: laringe eletrônica; Fonte: Autores.

É possível observar que três estudos (Almeida, 2017; Day \& Doyle, 2010; Eadie \& Doyle, 2004) abordaram a respeito da qualidade de vida apenas em falantes por prótese traqueoesofágica, um (Cox \& Doyle, 2014) referente a falantes apenas por laringe eletrônica, um (Correia et al., 2016) relacionou falantes por prótese traqueoesofágica e usuários de laringe eletrônica e seis (Carr et al., 2000; Eadie et al., 2013; Eadie \& Bowker, 2012; Farrand \& Duncan, 2007; Law et al., 2009; Moukarbel et al., 2011) fizeram a correlação com os três métodos: prótese traqueoesofágica, voz esofágica e laringe eletrônica.

O protocolo mais utilizado foi o Protocolo Qualidade de Vida Em Voz - QVV, citado em seis artigos (Almeida, 2017; Correia et al., 2016; Cox \& Doyle, 2014; Day \& Doyle, 2010; Eadie \& Bowker, 2012; Moukarbel et al., 2011) e seus resultados estão apresentados no Quadro 1. O protocolo University of Washington Quality of Life Questionnaire (UW-QOL) foi utilizado em três artigos (Almeida, 2017; Eadie et al., 2013; Eadie \& Bowker, 2012) (tabela 3).

Os protocolos EORTC Quality of Life Questionnaire (EORTC QLQ-C30) (Carr et al., 2000), Instrumento Qualidade De Vida da Universidade De Michigan Head And Neck -HNQOL (Eadie \& Doyle, 2004), Questionário de Qualidade de Vida SF-36 (Farrand \& Duncan, 2007) e o Questionário De Participação Após Laringectomia (CAPAL) (Law et al., 2009) foram citados uma vez cada, cujos resultados estão apresentados na Tabela 3. 
Tabela 3: Descrição dos artigos quanto ao método de comunicação, protocolo de qualidade de vida utilizado e resultados encontrados.

\begin{tabular}{|c|c|c|c|c|c|}
\hline Autor & Método de comunicação & $\mathbf{N}^{\mathbf{o}}$ & $\%$ & Protocolo QV & $\begin{array}{c}\text { Média QV para comunicação ou } \\
\text { global* }^{*}\end{array}$ \\
\hline $\begin{array}{l}\text { DAY, A. M.; DOYLE, Philip C } \\
(2010)^{14}\end{array}$ & Somente PTE & \multirow{3}{*}{3} & \multirow{3}{*}{27,27} & QVV & $\begin{array}{l}\text { geral: } 73.13 \text {; homens } 73,33 \\
\text { mulheres: } 72,92\end{array}$ \\
\hline Almeida, ALC. (2017) & Somente PTE & & & QVV & 82.75 \\
\hline Eadie TL, Doyle PC. (2004) ${ }^{15}$ & Somente PTE & & & HNQOL. 10 & 84.60 \\
\hline Cox SR, Doyle PC (2014) & Somente LE & 1 & 9,09 & QVV & homens: 65,$2 ;$ mulheres: 57,7 \\
\hline Correia, ME. (2016) & PTE X LE & 1 & 9,09 & QVV & PTE: 85,0; LE 50,05 \\
\hline Moukarbel, R (2011) ${ }^{23}$ & PTE X VE X LE & \multirow{6}{*}{6} & \multirow{6}{*}{54,54} & QVV & PTE: 76.5; VE: 69.7; LE: 53.5; \\
\hline Eadie TL, Bowker BC $(2012)^{20}$ & PTE X VE X LE & & & $\begin{array}{l}\text { UW-QOL } \\
\text { V-RQOL }\end{array}$ & $\begin{array}{l}\text { QVV: PTE: 66,45; VE: 72,94; LE: } \\
\text { 77,28/ UW-QOL:68,66* }\end{array}$ \\
\hline $\begin{array}{l}\text { Eadie TL, Day AMB, Sawin DE, } \\
\text { Lamvik K, Doyle PC. }(2013)^{18}\end{array}$ & PTE X VE X LE & & & UW-QOL & $\begin{array}{l}\text { PTE: 75,80; VE: } 100,00 ; \text { LE: } \\
62,14 ;\end{array}$ \\
\hline $\begin{array}{c}\text { Carr MM, Schmidbauer JA, Ma C, } \\
\text { Majaess L.(2000) }\end{array}$ & PTE X VE X LE & & & $\begin{array}{l}\text { EORTC QLQ- } \\
\quad \text { C30 }\end{array}$ & PTE: 63,33; VE: 86,36; LE: 69,70; \\
\hline Farrand P, Duncan F. (2007) ${ }^{21}$ & PTE X VE X LE & & & SF-36 & $\begin{array}{c}\text { PTE: 58,11; VE: 52,22; LE: } \\
50,43 *\end{array}$ \\
\hline Law IKY, Ma EPM, Yiu EML (2009) & PTE X VE X LE & & & CAPAL & $\begin{array}{c}\text { PTE: 27,92; VE: 43,57; LE: } \\
64,78\end{array}$ \\
\hline
\end{tabular}

Fonte: Autores.

\section{Discussão}

Os resultados obtidos no presente estudo apontam para um maior uso do instrumento QVV para avaliação da qualidade de vida em laringectomizados totais, seguido do University of Washington Quality of Life Questionnaire (UWQOL), utilizado em três artigos (Almeida, 2017; Eadie et al., 2013; Eadie \& Bowker, 2012), e dos protocolos EORTC Quality of Life Questionnaire (EORTC QLQ-C30), Instrumento Qualidade De Vida da Universidade De Michigan Head And Neck (HNQOL), Questionário de Qualidade de Vida -SF-36.

Durante a pesquisa foi possível observar que a maioria dos estudos são do Canadá e dos Estados Unidos, mas com os mesmos pesquisadores, podendo ser justificado por uma linha de pesquisa dos autores, no qual tem interesse em estudar a qualidade de vida de pacientes laringectomizados frente ao seu método de comunicação em diferentes países.

Os estudos incluídos foram publicados entre os anos 2000 a 2017. Sendo assim, nota -se uma falta de pesquisas recentes sobre o tema, havendo necessidade de mais estudos, já que a população laringectomizada total tem um grande impacto pós-cirúrgico que é a ausência da emissão vocal e o comprometimento da comunicação oral. O que afeta diretamente a qualidade de vida dos mesmos, devendo assim analisar qual método de comunicação alaríngea tem menor impacto na vida desses indivíduos.

Com relação ao impacto da voz alaríngea e os questionários encontrados, o QVV foi o protocolo mais utilizado nos estudos, apesar de não ser um instrumento calibrado para a população laringectomizada total, seus itens propiciam avaliar aspectos da voz e disfonia que impactam na qualidade de vida do indivíduo, tendo como foco exclusivo, a comunicação.

Assim, o QVV não mensura outros aspectos impactados pela remoção cirúrgica da laringe, como a deglutição, respiração e mudança na imagem, que são aspectos importantes a serem analisados na qualidade de vida do laringectomizado total, mas pode ser justificado sua grande utilização, devido a seu objetivo de analisar o impacto da disfonia na qualidade de vida do paciente, sendo que responde melhor a pergunta do presente estudo.

O QVV é um instrumento que avalia o impacto da disfonia na qualidade de vida de forma geral (Oliveira et al., 2013) foi o mais observado no presente estudo. Entretanto, os protocolos University of Washington-Quality of Life Questionnaire 
(UW-QOL); Functional Assessment of Cancer Therapy (FACT-H\&N); European Organization for Research and Treatment of Cancer (EORTC-C30/H\&N35) foram considerados os questionários mais utilizados para avaliação da QV em pacientes com Câncer de cabeça e pescoço, pela Conferência Internacional sobre Qualidade de Vida realizado na Virginia, EUA, em outubro de 2002 (Lima et al., 2011). Talvez, o uso destes protocolos seja maior devido a necessidade de se observar o impacto de outros aspectos na qualidade de vida, como a imagem, deglutição, respiração, além da comunicação. No caso do QVV, o único objetivo é verificar o impacto da alteração vocal na qualidade de vida e, talvez, seja o que melhor responda à pergunta do presente estudo.

O questionário V-RQOL, o mais utilizado nos artigos selecionados, foi desenvolvido em 1999 por Hogikyan et al (Hogikyan \& Sethuraman, 1999) para a língua inglesa e traduzido para diversas línguas e culturas (Contreras et al., 2019; Deshpande et al., 2009; Gasparini \& Behlau, 2009).

Os estudos (Almeida, 2017; Day \& Doyle, 2010) utilizaram o QVV para avaliar a qualidade de vida apenas em falantes por prótese traqueoesofágica, no qual foi possível notar que a média global foi respectivamente 73,13 e 82,75, sendo uma média mais próxima do escore 100 que é o melhor resultado, sendo assim nesses estudos foi possível notar que falantes por prótese traqueoesofágica apresentam uma qualidade de vida de grau bom, é o que corrobora com o estudo (Eadie \& Doyle, 2004) que avaliou apenas falantes por prótese traqueoesofágica, através dos domínios do HNQOL, que é um instrumento de qualidade de vida específico para pacientes com câncer de cabeça e pescoço e observou resultado para o domínio da comunicação de 84,60 pontos, sugerindo uma boa qualidade de vida para falantes por voz traqueoesofágica.

Estes resultados diferem do observado por Eadie, Bowker (Eadie \& Bowker, 2012), que comparou os três métodos de comunicação alaringea: prótese traqueoesofágica, voz esofágica e laringe eletrônica. Observaram que os falantes por prótese traqueoesofágica obtiveram a média global mais baixa 66,45, a voz esofágica em segundo lugar com 72,94 e a laringe eletrônica com a melhor média 77,27. Os autores definiram sua amostra de laringectomizados totais com pelo menos 9 meses de pós-operatório e não informaram se os laringectomizados de prótese traqueoesofágica apresentaram algum grau de dificuldade com a aquisição e desenvolvimento da voz, como também não informaram se algum dos pacientes havia sido submetido as cirurgias mais avançadas.

Outros pesquisadores (Cox \& Doyle, 2014) avaliaram a qualidade vida em pacientes usuários de laringe eletrônica e obseravam índices 65,2 em homens e 57,7 em mulheres, considerados bons. No entanto, ao comparar os resultados aqueles obtidos em estudos que avaliaram falantes por prótese traqueoesofagica, é possível observar que seus índices são piores. É o que demonstra o estudo de Correia e colaboradores (2016), no qual falantes por prótese traqueoesofágia apresentaram a média global de 85, já os usuários de laringe eletrônica obtiveram como média global 50,05, sendo possível observar que os falantes por prótese traqueoesofágica também apresentaram melhor qualidade de vida que os usuários de laringe eletrônica.

O estudo (Moukarbel et al., 2011) comparou os três métodos de comunicação e observaram que falantes por prótese traqueoesofágica obtiveram média global melhor, de 76,5, seguido por voz esofágica com 69,4 e por último a laringe eletrônica com 53,3. É o que também demonstrou Farrand e Duncan (Farrand \& Duncan, 2007) que utilizaram o Questionário de Qualidade de Vida - SF -36, que é uma medida genérica de qualidade de vida de autorrelato de 36 itens que consiste em oito dimensões. Observaram que falantes por prótese traqueoesofágica apresentaram melhores médias na categoria de percepção geral de saúde com score de 58,11, seguido por falantes por voz esofágica com 52,22 e por fins usuários de laringe eletrônica com 50,43.

O protocolo University of Washington Quality of Life Questionnaire - UWQOL foi utilizado, em três artigos, para avaliar o impacto da comunicação na qualidade de vida em laringectomizados totais. Almeida (Almeida, 2017) confirma que falantes por prótese traqueoesofagica apresentam bons índices de qualidade de vida com score 83.40 através do Protocolo University Of Washington Quality Of Life Questionnaire (UW-QOL). 
O estudo Eadie et al (Eadie et al., 2013) compara três métodos de comunicação alarígea, no qual falantes por voz esofágica apresentaram melhor média global, com 100 pontos, seguido por aqueles que utilizavam a prótese traqueoesofágica com 70,67 e por último, com escore de 62,86, usuários de laringe eletrônica, o que corrobora Carr et al (Carr et al., 2000), que utilizram a o Protocolo EORTC- Quality of Life Questionnaire (EORTC QLQ-C30) que é um sistema integrado para avaliar a qualidade de vida $(\mathrm{QV})$ de pacientes com câncer e pode ser utilizado em estudos de ensaios clínicos e outros tipos de pesquisa em que os resultados relatados pelo paciente são coletados, sendo que ele ressalta que falantes por voz esofágica apresentam melhor média na categoria de estado de saúde global com score de 63,33, seguido por usuários de laringe eletronica com 59,80 e por fins falantes por prótese traqueoesofagica com 46,67. Ambos os artigos colocam a voz esofágica como melhor método, o que diferencia é que no primeiro coloca a laringe eletrônica como o pior método e já no segundo artigo coloca falantes por prótese traqueoesofagica.

Em contrapartida, em um outro estudo (Law et al., 2009) comparou-se os três métodos de comunicação alaringea: prótese traqueoesofágica, voz esofágica e laringe eletrônica, esse artigo ultizou o Questionário de participação após laringectomia (CAPAL), no qual foi desenvolvido usando a Classificação Internacional de Funcionalidade, Incapacidade e Saúde (CIF) que avalia a qualidade de vida relacionada à comunicação em indivíduos que se submeteram a laringectomia e experimentaram 3 níveis de deficiência, a saber, fala deficiente, limitação da atividade de comunicação e restrição de participação na comunicação. Esse estudo obteve como resultado que os falantes por laringe eletrônica apresentaram melhor média total com score de 64,78, seguido por falantes de voz esofágica com 43,57 e por fim, falantes por prótese traqueoesofagica com 27,92.

O presente artigo também identificou que homens laringectomizados total têm menor impacto na qualidade de vida quando comparado às mulheres (Cox \& Doyle, 2014; Day \& Doyle, 2010), tanto para falantes por meio de prótese traqueoesofágica (Day \& Doyle, 2010), quanto para laringe eletrônica (Cox \& Doyle, 2014). Outro fato observado foi que estudos que avaliaram a comunicação apenas por meio da prótese traqueoesofágica apresentavam escores altos, próximos a 100 pontos (Almeida, 2017; Cox \& Doyle, 2014; Day \& Doyle, 2010; Law et al., 2009), diferente daqueles que a comparavam a outros métodos de comunicação alaríngea (Carr et al., 2000; Eadie \& Bowker, 2012; Law et al., 2009).

A laringe eletrônica apresentou piores índices de qualidade de vida na maioria dos estudos (Correia et al., 2016; Eadie et al., 2013; Farrand \& Duncan, 2007). Contrariamente, outros estudos (Eadie \& Bowker, 2012; Law et al., 2009) colocaram a laringe eletrônica como o grupo que mais se aproxima dos 100 pontos. Quanto à voz esofágica, essa foi considerada como um método de voz intermediário por outros estudos analisados.

Dessa forma, é possível observar que a prótese traqueoesofagica apresentou melhores índices na maioria dos artigos, o que corrobora com o estudo de Serra (Serra et al., 2015) no qual ressalta que atualmente a voz traqueoesofágica é o padrão ouro sobre os outros metodos de comunicação alaríngea. A voz esofágica apresentou media moderada de qualidade de vida, podendo ser justicado por apresentar como desvantagens o tempo de aprendizado e a variação no sucesso terapêutico, podendo ser $25 \%$ a até $70 \%$ (C. Furia et al., 2000). Por fim, a laringe eletrônica como método de pior impacto na qualidade de vida, podendo ser justificado por ser considerada uma voz robotizada e ter perdas de sonoridade (Davatz, 2011).

Foi possível observar que homens tem melhores índices de qualidade de vida quando comparado as mulheres. Dois estudos (Cox \& Doyle, 2014; Day \& Doyle, 2010) observaram melhores índices de qualidade de vida para homens quando comparado às mulheres. Possivelmente, pode ser justificado devido à frequência vocal estar mais próxima da faixa esperada para o sexo masculino.

Nessa revisão de escopo, foi observado uma falta de padronização a respeito do protocolo de qualidade de vida em pacientes larigenctomizados totais, no qual seria de suma importância um questionário de qualidade de vida voltado para as individualidades dessa população. Também foi observada a falta de estudos que abordassem exclusivamente a qualidade de 
vida em falantes por voz esofágica, já que para os outros dois métodos apresentaram artigos que explanaram individualmente o método e a sua qualidade de vida.

\section{Conclusão}

A presente revisão aponta que a os instrumentos mais utilizados para avaliar a qualidade de vida em indivíduos laringectomizados totais falantes por meio de comunicação alaríngea são: Qualidade de Vida em Voz (QVV), seguido do University of Washington Quality of Life Questionnaire (UW-QOL), e dos protocolos EORTC Quality of Life Questionnaire (EORTC QLQ-C30), Instrumento Qualidade De Vida da Universidade De Michigan Head And Neck (HNQOL), Questionário de Qualidade de Vida -SF-36.

De uma forma geral, a prótese traqueoesofágica apresentou melhores índices de qualidade de vida quando comparados a voz esofágica e laringe eletrônica. Apesar de não ter sido objetivo desse estudo, também foi possível observar que os artigos que fizeram comparações de gênero, verificaram que existe pouca diferença entre os gêneros, mas que há melhores índices de qualidades de vida para homens, quando comparado a mulheres.

\section{Referências}

Almeida, A. (2017). Qualidade de voz e vida pré e pós colocação de prótese traqueoesofágica. http://bdtd.ibict.br/vufind/Record/CAMP_c43dfb361bfef956aec590efa62f6d1f

Ana Carolina Ghirardi. (2007). Laringectomizados Usuários de Prótese Traqueoesofágica: Princípios e Métodos da Prática Fonoaudiológica.

Behlau, M. (2005). Voz: o livro do especialista. Revinter.

Blom, E. D., Singer, M. I., \& Hamaker, R. C. (1986). A Prospective Study of Tracheoesophageal Speech. Archives of Otolaryngology-Head \& Neck Surgery, 112(4), 440-447. https://doi.org/10.1001/ARCHOTOL.1986.03780040080017

Carr, M. M., Schmidbauer, J. A., Majaess, L., \& Smith, R. L. (2000). Communication after laryngectomy: an assessment of quality of life. Otolaryngology-Head and Neck Surgery: Official Journal of American Academy of Otolaryngology-Head and Neck Surgery, 122(1), 39-43. https://doi.org/10.1016/S01945998(00)70141-0

Choi, H. S., Park, Y. J., Lee, S. M., \& Kim, K. M. (2001). Functional Characteristics of a New Electrolarynx "Evada" Having a Force Sensing Resistor Sensor. Journal of Voice, 15(4), 592-599. https://doi.org/10.1016/S0892-1997(01)00062-5

CONITEC. (2018). Laringe eletrônica para reabilitação vocal de pacientes submetidos à laringectomia total. http://conitec.gov.br

Contreras, F., Gonzalez, N., Vivero, M., \& Guzman, M. (2019). Cross-cultural adaptation of the Chilean Version of the Voice-Related Quality of Life (VRQOL). CoDAS, 31(3). https://doi.org/10.1590/2317-1782/20192018213

Correia, M. E., Vianna, K. M. de P., \& Ghirardi, A. C. de A. M. (2016). Voz e qualidade de vida de laringectomizados totais: um estudo comparativo. Revista CEFAC, 18(4), 923-931. https://doi.org/10.1590/1982-0216201618423115

Cox, S. R., \& Doyle, P. C. (2014). The influence of electrolarynx use on postlaryngectomy voice-related quality of life. Otolaryngology - Head and Neck Surgery (United States), 150(6), 1005-1009. https://doi.org/10.1177/0194599814524704

Davatz, G. C. (2011). Reabilitação vocal e qualidade de vida em laringectomizados totais - PDF Download grátis [Universidade de São Paulo ]. https://docplayer.com.br/75941981-Reabilitacao-vocal-e-qualidade-de-vida-em-laringectomizados-totais.html.

Day, A., \& Doyle, P. (2010). Assessing self-reported measures of voice disability in tracheoesophageal speakers -. J Otolaryngol - Head Neck Surg, 39(6), 762-768. https://pubmed.ncbi.nlm.nih.gov/21144376/

Deshpande, M. S., Kakade, A. C., Chaukar, D. A., Gore, V. T., Pai, P. S., Chaturvedi, P., \& D’Cruz, A. K. (2009). Validation and assessment of voice-related quality of life in Indian patients undergoing total laryngectomy and primary tracheoesophageal puncture. Head \& Neck, 31(1), 37-44. https://doi.org/10.1002/HED.20922

Eadie, T. L., \& Bowker, B. C. (2012). Coping and quality of life after total laryngectomy. Otolaryngology - Head and Neck Surgery (United States), 146(6), 959-965. https://doi.org/10.1177/0194599812437315

Eadie, T. L., Day, A. M. B., Sawin, D. E., Lamvik, K., \& Doyle, P. C. (2013). Auditory-perceptual speech outcomes and quality of life after total laryngectomy. Otolaryngology - Head and Neck Surgery (United States), 148(1), 82-88. https://doi.org/10.1177/0194599812461755

Eadie, T. L., \& Doyle, P. C. (2004). Auditory-Perceptual Scaling and Quality of Life in Tracheoesophageal Speakers. The Laryngoscope, 114(4), 753-759. https://doi.org/10.1097/00005537-200404000-00030

Farrand, P., \& Duncan, F. (2007). Generic health-related quality of life amongst patients employing different voice restoration methods following total 
Research, Society and Development, v. 11, n. 2, e55011225883, 2022

(CC BY 4.0) | ISSN 2525-3409 | DOI: http://dx.doi.org/10.33448/rsd-v11i2.25883

laryngectomy. Psychology, Health and Medicine, 12(3), 255-265. https://doi.org/10.1080/13548500600665542

Furia, C., Mourão, L., \& Angelis, E. (2000). Reabilitação fonoaudiológica das laringectomias totais. In C. M. L. K. L. Furia (Ed.), A atualização da Fonoaudiologia no câncer de cabeça e pescoço. 1, 227-238. Lovise.

Gasparini, G., \& Behlau, M. (2009). Quality of Life: Validation of the Brazilian Version of the Voice-Related Quality of Life (V-RQOL) Measure. Journal of Voice, 23(1), 76-81. https://doi.org/10.1016/j.jvoice.2007.04.005

Hogikyan, N. D., \& Sethuraman, G. (1999). Validation of an instrument to measure voice-related quality of life (V-RQOL). Journal of Voice, 13(4), 557-569. https://doi.org/10.1016/S0892-1997(99)80010-1

Law, I. K. Y., Ma, E. P. M., \& Yiu, E. M. L. (2009). Speech intelligibility, acceptability, and communication-related quality of life in Chinese alaryngeal speakers. Archives of Otolaryngology--Head \& Neck Surgery, 135(7), 704-711. https://doi.org/10.1001/ARCHOTO.2009.71

Lima, M. A. G. de, Barbosa, L. N. F., \& Sougey, E. B. (2011). Avaliação do impacto na qualidade de vida em pacientes com câncer de laringe. Revista Da SBPH, 14(1), 18-40. http://pepsic.bvsalud.org/scielo.php?script=sci_arttext\&pid=S1516-08582011000100003\&lng=pt\&nrm=iso\&tlng=pt

Medeiros, N. C. (2008). Fala traqueoesofágica em laringectomizados totais: expressividade como proposta de intervenção. Dissertação de Mestrado. Pontifica Universidade Catolica de São Paulo. São Paulo, SP, Brasil.

Mombaque dos Santos, W., Regina Secoli, S., \& Alves de Araújo Püschel, V. (2018). Como citar este artigo A abordagem do Joanna Briggs Institute para revisões sistemáticas. https://doi.org/10.1590/1518-8345.2885.3074

Moukarbel, R. V., Doyle, P. C., Yoo, J. H., Franklin, J. H., Day, A. M. B., \& Fung, K. (2011). Voice-related quality of life (V-RQOL) outcomes in laryngectomees. Head \& Neck, 33(1), 31-36. https://doi.org/10.1002/HED.21409

Oliveira, I., Augusti, A., \& Siqueira, D. (2013). Avaliação de voz e qualidade de vida após laringectomia supracricóide Avaliação de voz e qualidade de vida após laringectomia supracricóide. Audiol - Commun Res., 18(4), 353-60. https://www.scielo.br/j/acr/a/DBsrprnFTJvgXqHvJYXTDMC/?lang=pt

Serra, A., Di Mauro, P., Spataro, D., Maiolino, L., \& Cocuzza, S. (2015). Riabilitazione vocale post laringectomia con voce protesica: 15 anni di esperienza della Clinica Otorinolaringoiatrica dell'Università di Catania. Analisi retrospettiva dei dati e revisione della letteratura. Acta Otorhinolaryngologica Italica, 35(6), 412-419. https://doi.org/10.14639/0392-100X-680

Soto, N. C., Teles, L., \& Fukuyama. (2005). Avaliação Perceptivo-Auditiva E acústica Perceptual and acoustic evaluation of the. Revista CEFAC, 7(4), 496502. 